\title{
Genetic and phenotypic characterisation of inherited myopathies in a tertiary neuromuscular centre
}

Enrico Bugiardini, ${ }^{\mathrm{a}, \mathrm{b}}$ Alaa Khan, ${ }^{\mathrm{b}}$ Rahul Phadke, ${ }^{\mathrm{c}, \mathrm{d}}$ David S. Lynch, ${ }^{\mathrm{b}}$ Andrea Cortese,${ }^{\mathrm{a}, \mathrm{b}}$ Lucy Feng, ${ }^{c}$ Qiang Gang, ${ }^{\text {a,b }}$ Alan M. Pittman, ${ }^{\mathrm{b}, \mathrm{e}}$ Jasper M. Morrow, ${ }^{\mathrm{a}}$ Chris Turner, ${ }^{\mathrm{a}}$ Aisling S. Carr, ${ }^{a}$ Ros Quinlivan, ${ }^{a}$ Alexander M Rossor, ${ }^{\mathrm{a}, \mathrm{b}}$ Janice Holton, ${ }^{\mathrm{a}, \mathrm{d}}$ Matt Parton, ${ }^{\mathrm{a}}$ Julian C Blake, ${ }^{\mathrm{a}}$ Mary M Reilly, ${ }^{a, b}$ Henry Houlden, ${ }^{b}$ Emma Matthews, ${ }^{a, b}$ Michael G. Hanna ${ }^{a, b}$

\section{Corresponding author}

Professor Michael G Hanna

Box 102, National Hospital for Neurology and Neurosurgery, Queen Square, London WC1N 3BG, UK. Tel.: +44 02034483014 email: m.hanna@ucl.ac.uk

\section{Affiliations}

aMRC Centre for Neuromuscular Diseases, UCL Institute of Neurology and National Hospital for Neurology and Neurosurgery, Queen Square, London, UK

bDepartment of Neuromuscular Diseases, UCL Institute of Neurology, Queen Square, London, UK

'Division of Neuropathology, Dubowitz Neuromuscular Centre, UCL and Great Ormond Street Hospital for Children, UK

dDivision of Neuropathology, National Hospital for Neurology and Neurosurgery, Queen Square, London, UK

eSt George's, University of London, Cranmer Terrace, London, UK 


\section{ABSTRACT}

Diagnosis of inherited myopathies can be a challenging and lengthy process due to broad genetic and phenotypic heterogeneity. In this study we applied focused exome sequencing to investigate a cohort of 100 complex adult myopathy cases who remained undiagnosed despite extensive investigation. We evaluated the frequency of genetic diagnoses, clinical and pathological factors most likely to be associated with a positive diagnosis, clinical pitfalls and new phenotypic insights that could help to guide future clinical practice. We identified pathogenic/likely pathogenic variants in 32/100 cases. TTN-related myopathy was the most common diagnosis (4/32 cases) but the majority of positive diagnoses related to a single gene each. Childhood onset of symptoms was more likely to be associated with a positive diagnosis. Atypical and new clinico-pathological phenotypes with diagnostic pitfalls were identified. These include the new emerging group of neuromyopathy genes (HSPB1, BICD2) and atypical biopsy findings: COL6A-related myopathy with mitochondrial features, DOK7 presenting as myopathy with minicores and DES-related myopathy without myofibrillar pathology. Our data demonstrates the diagnostic efficacy of broad NGS screening when combined with detailed clinico-pathological phenotyping in a complex neuromuscular cohort. Atypical clinico-pathological features may delay the diagnostic process if smaller targeted gene panels are used.

Keywords: Inherited myopathies; Next generation sequencing; Molecular diagnosis; Distal myopathy. 


\section{INTRODUCTION}

Inherited myopathies are a heterogeneous group of disabling diseases characterized by muscle weakness with or without additional cardiac and respiratory impairment. The classification of inherited myopathies is challenging given the broad heterogeneity of the clinical, pathological and genetic features. Classifications have been attempted using the pattern of weakness and underlying protein or genetic defect. However from a genetic point of view it is becoming increasingly clear that different entities overlap in their clinical and pathological manifestations. Until recently the diagnostic approach for inherited myopathies used a combination of clinical assessment and muscle biopsy analysis to guide Sanger sequencing of individual candidate genes. However, more than 150 genes have been associated with myopathy [1]. Moreover mutations in the same gene can cause different phenotypes and conversely different genes can cause the same phenotype. To further complicate the genetic diagnosis, several genes causing myopathies are among the largest human genes [2]. Sanger sequencing is consequently time consuming and expensive and as a result "gate keeping" is often applied to diagnostic requests with atypical or unusual clinical features.

The advent of next generation sequencing (NGS) has revolutionised the diagnostic approach for inherited myopathies allowing the screening of multiple genes in parallel. NGS diagnostic rate in myopathies varies broadly (between 16 and $65 \%$ of cases) depending on the cohort investigated and the NGS approach used [3-5]. NGS has also been pivotal for recognising new genotype-phenotype correlations. In clinical practice however, NGS is largely limited to small disease specific panels and it can still be difficult or costly to obtain testing outside of a research environment for atypical cases. 
In this study we applied focused exome sequencing to investigate a cohort of 100 complex adult cases with undiagnosed myopathy despite extensive investigation who were referred to our tertiary diagnostic centre. We evaluated the frequency of genetic diagnoses, clinical pathological factors most likely to be associated with a positive diagnosis and detail new phenotypic insights that help to guide future clinical practise.

\section{METHODS}

\subsection{Patient cohort, data collection and statistical analysis}

We investigated 100 adult patients referred to us at the MRC Centre for Neuromuscular Diseases, London, with a clinical diagnosis of inherited myopathy but no genetic diagnosis despite extensive clinical, radiological and pathological investigations. Previously, genetic tests were performed using a candidate gene approach based on the results of the above investigations.

Clinical, neurophysiological, pathological and radiological data were retrieved from the clinical notes. We classified the patients based on age of onset: childhood onset $(0-<10$ years), juvenile/adult onset (10-39), and late onset $(\geq 40)$. We also grouped clinical, neurophysiological, pathological and MRI features by categorical variable. For clinical features based on predominant pattern of weakness we considered: predominant proximal weakness, predominant distal weakness and other phenotypes. The latter includes cases with marked extraocular muscle involvement (ophthalmoparesis), axial myopathies, scapulo-peroneal myopathies and more complex phenotypes. We classified neurophysiology results into general categories of: normal, myopathic, neuropathic and mixed. Muscle biopsy results were categorised as histologically normal/minimal changes, end stage, neurogenic, or 
myopathic. In the latter we also recorded predominant pathological findings: general dystrophic features, general myopathic features, protein aggregation and/or rimmed vacuoles, core-like lesions, tubular aggregates, nemaline bodies, mitochondrial features. Finally, for muscle MRI we considered the presence of a normal or abnormal signal in muscle (fatty infiltration in T1). If abnormal, we then evaluated if muscle involvement was proximal (thigh), distal or both. Statistical analyses were performed using SPSS version 24.0. (Armonk, NY: IBM Corp.). Fisher Exact test was used to evaluate association between genetic diagnosis and clinical, neurophysiological, pathological and radiological categorical data. Variable with three categories (age of onset, phenotype) were tested using Pearson Chi Square. P-values $<0.05$ were considered to be statistically significant. Informed consent for genetic testing was obtained from all patients and the study was approved and performed under the ethical guidelines issued by our institution.

\subsection{Focused Exome Sequencing and Sanger sequencing}

Focused exome sequencing was performed using the SureSelect Focused Exome (Agilent), according to the manufacturer's protocol. Filtering of variants was performed using VarAft platform [6]. We screened a list of 322 genes related to neuromuscular disease and hereditary cardiomyopathies reported in the Neuromuscular gene table 2016 (http://www.musclegenetable.fr). First we filtered out variants with an allele frequency higher than 1\% in ExAC, 1000 Genome or ESP6500 database. We excluded all synonymous and deep intronic variants. Remaining variants were then classified using American College of Medical Genetics (ACMG) criteria [7] and those deemed likely or definitely pathogenic were confirmed using Sanger sequencing. 


\section{RESULTS}

\subsection{Overall results}

We recruited 100 patients attending the muscle specialist service at the MRC Centre for Neuromuscular Diseases (London). Focused exome sequencing metric showed that on average $95 \%$ of targeted bases were read at least 10 times.

In our cohort the mean age at the time of the study was 54.8 years (STD=15.9, range $21-87$ ), and there was a slight male prevalence (54\%). Main clinical and pathological features are reported in Table 1.

We identified pathogenic or likely pathogenic variants in $32 / 100$ cases. $59 \%$ of these variants were already reported in the literature as causing neuromuscular disorders. TTN-related myopathy was the most common genetic diagnosis (4/32 cases) presenting with either juvenile onset distal myopathy with rimmed vacuoles $(n=2)$ or congenital myopathy $(n=2)$. The majority of remaining diagnoses were related to a single gene each. In nine patients we found genes associated with congenital myopathy/muscular dystrophies $($ ACTA1 $\mathrm{n}=1 ; \operatorname{COL6A1} \mathrm{n}=2 ; \operatorname{COL6A3} \mathrm{n}=1 ; M Y H 7$ $\mathrm{n}=2 ;$ SEPN1 $\mathrm{n}=2 ; \operatorname{TPM} 3 \mathrm{n}=1$ ), whereas genes reported as associated with Limb Girdle Muscular Dystrophy (LGMD) were found in five patients ( $A N O 5 \mathrm{n}=1 ; D E S$ (recessive) $\mathrm{n}=1 ; D N A J B 6 \mathrm{n}=2 ; G M P P B \mathrm{n}=1$ ). In remaining cases a mixture of genes associates with other myopathies such as myofibrillar, distal and tubular aggregates myopathies were found (GNE $\mathrm{n}=2 ; F H L 1 \mathrm{n}=1 ;$ ORAI1 $\mathrm{n}=1 ;$ MYOT=1; STIM1 $\mathrm{n}=2$; $V C P=1)$. In 5 cases, genes not typically associated with myopathy were identified including genes causing congenital myasthenia $(D O K 7=2)$ and inherited neuropathy 
(BICD2 $\mathrm{n}=1 ; H S P B 1 \mathrm{n}=1 ; \operatorname{MFN} 2 \mathrm{n}=1)$. In one case a disease mimicking a myopathy was found ( $F L N A=1)$ (see Supplementary Table 1 for details on individual cases). We identified pathogenic/likely pathogenic variants in 15/30 (50\%) of childhood onset cases, $12 / 34$ (35\%) of juvenile/adult onset cases and $5 / 36$ (14\%) of late onset cases. If considered by pattern of weakness, patients with proximal weakness were diagnosed in $14 / 47(30 \%)$ of cases, distal weakness $13 / 33(39 \%)$, complex and other phenotypes in 5/20 (25\%) cases (Figure 1A). In the muscle pathology subgroups patients with normal/minimal changes or general non-specific myopathic features had the lowest diagnostic rate (11 and 13\% respectively) (Figure 1B). Considering neurophysiology results, we did not identify any pathogenic/likely pathogenic variant in patients with normal EMG/NCS. In 21/65 (35\%) of patients with abnormal EMG/NCS we found a genetic diagnosis without main differences between subgroups (35\%, $20 \%$ and $38 \%$ for myopathic, neuropathic and mixed respectively). In MRI subgroups, in only one case with a normal scan we did reach a genetic diagnosis. Most of patients with abnormal MRI had an involvement of both proximal and distal muscles (Table 1) with an overall diagnostic hit rate of $32 \%$. We compared the characteristics of patients with a causative gene identified $(n=32)$ with those remaining without a genetic diagnosis $(n=68)$ (see Table 2). Childhood onset was associated with a positive diagnosis whereas late onset and normal EMG were more likely to be associated with a negative diagnosis.

\subsection{New and atypical genotype-phenotype correlations}

Among the 32 cases with pathogenic/likely pathogenic variants we identified several atypical and new phenotypes. A summary of the clinical and genetic details is 
reported in supplementary table 1 . Here we highlight clinical lessons from difficult cases with new clinical and diagnostic implications.

\subsubsection{Distal weakness - myopathy or motor neuropathy?}

In three cases of distal weakness we found variants in genes previously associated with motor neuropathy.

Case ID9 presented with distal weakness at age 19. A muscle biopsy performed early in the disease course was myopathic but an evolving neuropathic EMG pattern with evidence of axonal motor neuropathy was seen as the disease progressed which led to diagnostic confusion. NGS eventually demonstrated a homozygous HSPB1 variant (c.418C>G, p.Arg140Gly) that was previously reported in heterozygous cases of motor neuropathy. This case was reported in detail previously [8] as it illustrates a new phenotype of heat shock proteins causing both myopathy and motor neuropathy, an increasingly recognised phenomenon $[8,9]$.

A second case of late onset distal weakness with an absence of sensory symptoms and signs presented at age 43 (ID62). Clinically the patient was felt to have a distal myopathy. Nerve conduction studies demonstrated a length dependent predominantly motor neuropathy with minor sensory involvement but the needle EMG pattern was felt to be consistent with a myopathic process, both distally in the lower limbs and also in the proximal upper limb muscles. The features seen were of many low amplitude and brief motor units recruiting early. CK was $538 \mathrm{IU} / \mathrm{L}$. This conflicting picture prompted a muscle biopsy but only angular atrophic fibres and clustering of type I fibres compatible with denervation were seen. Repeat needle EMG later in the disease course was consistent with neurogenic change. NGS 
identified a previously reported heterozygous variant in MFN2 (c.311G>A, p.Arg104GIn).

In the third case (ID 40) the proband experienced neonatal hypotonia and congenital bilateral hip dislocations. He had delayed motor development walking at fifteen months but gait was never considered normal. During his childhood he had slowly progressive muscle weakness. At 44 years, clinical examination showed a scapuloperoneal pattern of muscle weakness. CK was mildly elevated to $279 \mathrm{IU} / \mathrm{L}$. A muscle biopsy at 27 years showed myopathy with tubular aggregates. The clinical neurophysiology consistently demonstrated normal motor and sensory nerve conduction without any features of peripheral neuropathy but needle EMG patterns were more equivocal showing, on several occasions, features of both myopathy (low amplitude and brief motor units) and motor neuropathy (large rapidly firing units). A further biopsy at 37 years demonstrated accentuated tubular aggregate pathology, further confirmed by electron microscopy (case 8 reported in Brady et al.[10]) A muscle MRI at 41 years old showed an abnormal pattern of muscle involvement typical of BICD2 related disease (Figure 2). With NGS we found a novel heterozygous variant in BICD2 (c.629A $>$ C; p.His210Pro) which occurs at a highly conserved amino acid in the dynein binding region of the protein in which pathogenic mutations have been previously described $[11,12]$.This diagnosis is compatible with much of the clinical presentation but not previously associated with tubular aggregates. A variant of uncertain significance (VUS) in the CASQ1 gene (c.1136T>C; p.lle379Thr) recently associated with tubular aggregate myopathy [13] was additionally identified in a parallel whole exome sequencing project.

\subsubsection{Unexpected biopsy findings}




\subsubsection{COL6A-related myopathy with prominent mitochondrial features}

We identified three cases (ID 36, 52, 67) with suspected COL6-related myopathy. All of them had onset in childhood of mild neck and proximal limb weakness, contractures, normal CK and myopathic EMG. In two cases however (ID 52 and ID 67) muscle biopsies showed prominent mitochondrial abnormalities (Figure 3) not typically associated with human COL6-myopathy. This prompted us to consider other or additional diagnoses complicating the diagnostic process. Further analysis of muscle tissue from ID 52 and ID 67 showed complex IV (CIV) deficiency (0.005 and $0.012 \mathrm{CIV} /$ citrate synthase activity respectively; normal values $>0.014$ ) but normal whole mtDNA analysis. NGS ultimately did confirm pathogenic variants in both patients (COL6A3 c.6220G $>A$ and COL6A1 c.1056+1G>A respectively in ID 52 and 67). Review of muscle MRI showed a pattern of muscle involvement considered typical for COL6-related myopathy (Figure 3C and F). Consequent collagen immunostaining was overall normal in the first case and showed a subtle patchy reduction in the second case (Figure 3G-L).

\subsubsection{Desminopathy with absence of desmin accumulation}

In another case (ID4) presenting with childhood onset limb girdle weakness we found a homozygous $D E S$ variant c. $1289-2 A>G$. The muscle biopsy at 34 years old showed marked myopathic changes with increase in fibre size, scattered regenerating fibres and frequent round atrophic fibres. There was no evidence of protein accumulation, generally considered the pathological hallmark of desmin related myopathies (Figure 4A-B) [14]. The most recent clinical examination at 45 years old demonstrated marked weakness predominantly in proximal muscles without evidence of cardiac involvement. NGS screening pinpointed the genetic 
defect in DES and as a consequence the muscle biopsy was stained for desmin. Uneven desmin labelling was found but without definite protein aggregation (Figure 4C-D).

\subsubsection{Congenital myasthenia presenting as proximal myopathy}

We identified two patients with mutations in the DOK7 gene usually associated with congenital myasthenic syndrome. One case (ID 35) presented as a child with delayed motor milestones, proximal weakness, scoliosis and mild elbow contractures. CK was normal. She had two muscle biopsies (at 5 and 23 years of age) that showed myopathic changes with preserved fibre typing and unevenness on oxidative stains in the first biopsy progressing to multiminicores and occasional larger cores in the second biopsy (Figure 4E-F). An EMG done at 34 years old was consistent with a mild proximal myopathy. Repetitive nerve stimulation was within normal limits and there was only a non-specific minimal increased jitter of the orbicularis oculi at single fibre EMG. Examination at 35-years-old showed mild ptosis, contractures at the shoulders and elbows, mild proximal weakness and scoliosis. MRI demonstrated mild streaky infiltration in most of the thigh and calf muscles bilaterally. Based on the biopsy findings we pursued a diagnostic panel of 23 genes associated with congenital myopathy but results were negative. Our broad NGS screening demonstrated two previously described DOK7 mutations (c.1120_1121insGCCT and c.1330_1331insCTGG).

The second case (ID 79) presented in later life with difficulty climbing stairs from 33 years of age although she retrospectively reported never having been able to run as fast as her peers in childhood. Her muscle biopsy showed myopathic features with increased variation in fibre size, internal nuclei and a minor degree of necrosis and 
regeneration. Clinical examination at 43-years-old showed minimal facial and proximal weakness.

\subsubsection{Myopathy mimics}

Case ID21 presented with contractures, stiffness and painful joints since childhood. An EMG done elsewhere was reported as myopathic which prompted referral to our centre at age 28 years. Due to additional diagnoses of mitral stenosis and aortic regurgitation requiring valve replacement and warfarin therapy at 26 years of age we elected not to repeat the EMG. He developed keloid scars after surgery. His mother reported stiffness of the joints and his maternal grandfather was said to be stiff with restricted movements. On clinical examination at 37 years old the joints were generally stiff and he had multiple contractures of the elbows, little and ring fingers in addition to minimal contracture of the knees and shoulders. The muscle biopsy slides were unfortunately not retrievable for review but were reported as abnormal with evidence of mitochondrial hyperplasia. Muscle MRI performed at our centre was normal. CK was $302 \mathrm{IU} / \mathrm{L}$. Ultimately we identified a mutation in FLNA (c.4726G>A; p.Gly1576Arg), a gene not routinely screened for myopathies.

\section{DISCUSSION}

\subsection{Overall results}

Next generation sequencing is gradually changing the diagnostic approach to neuromuscular disorders and is beginning to be considered as a first line approach in the paediatric setting [15]. Several reports have evaluated the efficacy of various NGS approaches in specific disease subgroups with variable diagnostic hit rates 
depending on methods used and cohort selected $[3,4,16]$. Our study reflects a real life heterogeneous group of complex and varied presentations to our tertiary neuromuscular centre that remained undiagnosed after the exclusion of common or typical cases; e.g. the commonest limb girdle muscular dystrophy (LGMD) (i.e. CAPN3) [17] was not found in this cohort at all as these cases were diagnosed by targeted gene sequencing.

Using NGS in our cohort of 100 complex unsolved cases we reached a genetic diagnosis in $32 \%$. The prevalence of the genes found is reported in table 3 . Our highest diagnostic rate $(13 / 33,39 \%)$ was in those presenting with distal weakness. Distal myopathies are a challenging group of diseases [18] and their proportion may be expected to increase in a cohort such as ours that has already been investigated for common causes of myopathy. Of note two cases with distal myopathies were diagnosed with GNE-related myopathy. The candidate gene approach failed to identify the diagnosis as muscle biopsy did not show rimmed vacuoles and consequently GNE specific testing was not requested. This is an increasingly recognized pitfall in GNE-related myopathy [19] and muscle MRI has been suggested as a useful diagnostic tool both for targeting the biopsy and for pattern analysis in distal myopathies [20]. Of note muscle MRI showed an involvement of proximal muscles in 24 of 27 (89\%) cases with MRI available further highlighting the importance of including thigh muscles scanning in distal myopathies.

TTN gene variants accounted for the largest single group of diagnoses (4/32 cases) although with divergent phenotypes (juvenile onset distal myopathy and congenital myopathy with cardiomyopathy). TTN is a huge gene with multiple clinical presentations making gate keeping and Sanger sequencing a challenge which may have contributed to the frequency of cases in our cohort. It is of note that across our 
32 positive cases, however, 22 genes were implicated with many accounting for only one individual case. This argues for the utility of a broad NGS approach in a cohort such as ours which has already had common or typical diagnoses excluded.

Features more likely to be associated with a genetic diagnosis included younger age of onset, abnormal MRI and/or EMG. MRI was abnormal in all cases with a genetic diagnosis except the case with FLNA mutation who ultimately probably did not have a myopathy.

In $68 \%$ of our cases we did not identify candidate variants satisfying the criteria for being pathogenic/likely pathogenic. This is a rate in keeping with other studies on NGS use in myopathy [16] especially if applied in a cohort already screened by single gene approach [21]. Of note most of the patients without a diagnosis were sporadic $(84 \%)$ and this limited the interpretation of some genetic variants. Other factors that may contribute to the negative results in this cohort are that we screened genes known to be associated with neuromuscular disease at the time of the project and it is possible that new genes identified since that timepoint may account for some of our cases. Furthermore complex genetic mechanisms, not explored by our analysis, could underlie some of the negative cases. These include repeat expansion diseases, copy number variants and deep intronic mutations [21]. Oligogenic inheritance has also been speculated as a potential pathogenic mechanism [22] but this has not been explored in this study.

\subsection{New and atypical genotype-phenotype correlations - clinical lessons}

\subsubsection{Neuromyopathy- an emerging entity}

We identified three cases with variants in genes previously associated with isolated motor neuropathies/neuronopathies (HSPB1, MFN2, BICD2). The differential 
diagnoses were complicated by a traditional approach of trying to separate the clinical entity into either nerve or muscle pathology. In two cases (HSPB1, BICD2) it was clear there was evidence of dual pathology. Mutations in small heat shock proteins have now been recognised to cause both a motor neuropathy and a myopathy [8,23]. Whilst BICD2 is also associated with dual pathology [24] no link has been established with tubular aggregates. Our case had many typical features of BICD2-disease but we also found a VUS in the CASQ1 gene. CASQ1 variants have recently been reported in tubular aggregate myopathies without neurogenic features so we cannot exclude the possibility of double-trouble in this case. In our third case, EMG initially caused confusion although a re-evaluation of the EMG later in the disease course ultimately determined a neuropathic process. All three of these cases illustrate the difficulties of interpretation of needle EMG. Routine needle EMG in clinical practice is largely a qualitative evaluation and is operator dependent. There are however some definite EMG characteristics that usually allow separation of patients into neurogenic or myopathic groups. Our case of MFN2 mutation initially had EMG features that were definitely myopathic but that evolved over time to the more usually expected neurogenic changes for mutations in this gene. Nerve conduction however always hinted at a length dependent peripheral neuropathy. Interpretation of the EMG in our case of BICD2 was less easy to categorise as it showed a mix of small myopathic motor units alongside large and fast firing neurogenic motor units both within and between muscles sampled. In complex cases of distal weakness such as these, a combined NGS approach examining both muscle and nerve genes associated with distal weakness is indicated.

\subsubsection{Unexpected biopsy findings}




\subsubsection{COL6A-related myopathy with mitochondrial features}

In two cases with COL6A-related myopathy we found prominent mitochondrial abnormalities in the muscle biopsy. Mitochondrial dysfunction has been reported in COL6-related diseases mainly linked to a dysregulation of the mitochondrial permeability transition pore [25] but definitive mitochondrial features in the muscle biopsy are not commonly reported in Bethlem myopathy [26, 27]. This initially led us to consider alternative or additional diagnoses including mitochondrial myopathy. Reevaluation of the clinical features and MRI findings however ascertained that these cases were otherwise typical for Bethlem myopathy and NGS confirmed this diagnosis. It may be that mitochondrial pathology becomes more apparent with increasing age as muscle biopsies in our cases were performed at age 49 and 57 while the majority reported in the literature are paediatric cases. A single case of Bethlem myopathy with COX negative fibres was reported in a 61-year-old patient [28]. Of note, both biopsies showed extensive fatty infiltration not usually seen in primary mitochondrial disease even in advanced stages. Furthermore it should be noted that collagen VI immunolabeling in skeletal muscle sections appears normal in most typical adult cases of Bethlem myopathy.

Our cases illustrate that mitochondrial pathology can be seen in adult Bethlem patients and should not deter from pursuing this diagnosis.

\subsubsection{Desminopathy with absence of desmin accumulation}

The DES case (ID4) is highlighted as the patient presented with a recessive limb girdle muscular dystrophy without myofibrillar pathology evident on muscle biopsy. This differs from the characteristic findings that would normally prompt $D E S$ gene analysis. A lack of such characteristic biopsy findings has been reported in a single 
family of the same ethnicity, carrying the same mutation and having similar pathology [29] which may suggest a genotype-phenotype correlation more than a sampling error. Irrespective these cases illustrate caution against excluding genetic testing based on a lack of typical pathology.

\subsubsection{Congenital myasthenia presenting as proximal myopathy}

$D O K 7$ typically causes congenital myasthenia but it is increasingly recognised that it may present as a limb girdle phenotype [30] without significant neuromuscular junction dysfunction seen on neurophysiological testing as was demonstrated by our two cases. This initially deterred us from requesting diagnostic testing for congenital myasthenia genes. In one of our cases the muscle biopsy demonstrated a disturbance of internal architecture suggesting minicore pathology. Core-like areas were reported in $43 \%$ of cases in one series of DOK7 related diseases [31] suggesting such findings should raise the possibility of DOK7 in the differential diagnosis. All the mutations found in our cases were previously reported in association with DOK7-related disease supporting pathogenicity. One of our patients gained subsequent benefit from salbutamol treatment highlighting the important treatment implications of a diagnosis of DOK7 related myopathy.

\subsubsection{Myopathy mimics}

The FLNA gene codes for the filamin A protein involved in several cell functions including cell signalling, cell motility and scaffolding [32]. Mutations in FLNA have been reported with a broad spectrum of clinical manifestations including 
periventricular nodular heterotopia, X-linked cardiac valvular dysplasia and otopalatodigital spectrum disorders.

Our case presented with an X-linked syndrome with multiple contracture, cardiac valvulopathy and keloid scarring. Keloid scarring is rare in FLNA associated disease but our case closely resembles another case with the same FLNA pathogenic variant (c.4726G>A; p.Gly1576Arg) [33] and prominent keloid scars. This supports an important genotype-phenotype association. The evidence for myopathy was based on investigations done elsewhere and due to confounding factors we did not repeat pertinent investigations but in a real world clinical setting similar cases may present to a neuromuscular clinic.

\section{CONCLUSIONS}

Our data demonstrates the diagnostic efficacy of broad NGS screening in a clinically and genetically heterogeneous complex neuromuscular cohort when combined with a multi-disciplinary assessment. Although a genetic diagnosis was achieved in many cases with extensive genetic analysis, a detailed case-by-case re-evaluation has been essential in the diagnostic process in our cohort. This is exemplified by the COL6A-related myopathy cases, in which muscle pathology with secondary mitochondrial dysfunction complicated the diagnostic process but a careful reevaluation of all factors in these cases including muscle MRI supported the identified COL6A1 and COL6A3 variants. NGS can enhance the detection of rare genetic disease or cases presenting with atypical phenotype but it can also generate multiple variants of uncertain significance that can complicate the diagnostic process. As NGS technologies become increasingly accessible, ancillary tests, detailed clinical evaluation and diagnostic biomarkers will be essential to support data analysis. Our 
study highlights the need for interpretation of NGS data through a multidisciplinary evaluation including clinical phenotyping and appraisal of muscle pathology, neurophysiology and muscle imaging. 


\section{ACKNOLEDGMENTS}

The authors would like to thank Darren Chambers and Lee Martindale for their technical assistance. The research leading to these results has received funding from the European Community's Seventh Framework Programme (FP7/2007-2013) under grant agreement n²012-305121 "Integrated European - omics research project for diagnosis and therapy in rare neuromuscular and neurodegenerative diseases (NEUROMICS)". This work is also supported by a Medical Research Council Centre grant (G0601943). Part of this work was undertaken in the University College London Hospitals/University College London Institute of Neurology sequencing facility, which received a proportion of funding from the Department of Health's National Institute for Health Research Biomedical Research Centres funding scheme. RP is supported by the HSS England Diagnostic and Advisory Service for congenital myopathies and congenital muscular dystrophies in London. AC is funded by the Inherited Neuropathy Consortium (INC), which is a part of the NIH Rare Diseases Clinical Research Network (RDCRN) (U54NS065712) and Wellcome Trust (204841/Z/16/Z) and MRC (MR/T001712/1). AMR is funded by a Wellcome Trust Postdoctoral Fellowship for Clinicians (110043/Z/15/Z). EM is funded by a Wellcome Trust Clinical Research Career Development Fellowship. MGH research is supported by an MRC Centre grant (MR/S005021/1) and by the UCLH NIHR Biomedical Research Centre. 


\section{REFERENCES}

[1] Kaplan JC, Hamroun D. The 2016 version of the gene table of monogenic neuromuscular disorders (nuclear genome). Neuromuscul Disord 2015;25:991-1020. doi:10.1016/i.nmd.2015.10.010.

[2] Laing NG. Genetics of neuromuscular disorders. Crit Rev Clin Lab Sci 2012;49:33-48. doi:10.3109/10408363.2012.658906.

[3] Ghaoui R, Cooper ST, Lek M, Jones K, Corbett A, Reddel SW, et al. Use of whole-exome sequencing for diagnosis of limb-girdle muscular dystrophy:

Outcomes and lessons learned. JAMA Neurol 2015.

doi:10.1001/jamaneurol.2015.2274.

[4] Kuhn M, Glaser D, Joshi PR, Zierz S, Wenninger S, Schoser B, et al. Utility of a next-generation sequencing-based gene panel investigation in German patients with genetically unclassified limb-girdle muscular dystrophy. J Neurol 2016;263:743-50. doi:10.1007/s00415-016-8036-0.

[5] Evila A, Arumilli M, Udd B, Hackman P. Targeted next-generation sequencing assay for detection of mutations in primary myopathies. Neuromuscul Disord 2016;26:7-15. doi:10.1016/i.nmd.2015.10.003.

[6] Desvignes JP, Bartoli M, Delague V, Krahn M, Miltgen M, Beroud C, et al. VarAFT: a variant annotation and filtration system for human next generation sequencing data. Nucleic Acids Res 2018;46:W545-53. doi:10.1093/nar/gky471.

[7] Richards S, Aziz N, Bale S, Bick D, Das S, Gastier-Foster J, et al. Standards and guidelines for the interpretation of sequence variants: a joint consensus recommendation of the American College of Medical Genetics and Genomics and the Association for Molecular Pathology. Genet Med 2015;17:405-24. 
doi:10.1038/gim.2015.30.

[8] Bugiardini E, Rossor AM, Lynch DS, Swash M, Pittman AM, Blake JC, et al. Homozygous mutation in HSPB1 causing distal vacuolar myopathy and motor neuropathy. Neurol Genet 2017;3. doi:10.1212/NXG.0000000000000168.

[9] Ghaoui R, Palmio J, Brewer J, Lek M, Needham M, Evila A, et al. Mutations in HSPB8 causing a new phenotype of distal myopathy and motor neuropathy. Neurology 2015. doi:10.1212/WNL.0000000000002324.

[10] Brady S, Healy EG, Gang Q, Parton M, Quinlivan R, Jacob S, et al. Tubular Aggregates and Cylindrical Spirals Have Distinct Immunohistochemical Signatures. J Neuropathol Exp Neurol 2016;75:1171-8. doi:10.1093/inen/nlw096.

[11] Oates EC, Rossor AM, Hafezparast M, Gonzalez M, Speziani F, MacArthur DG, et al. Mutations in BICD2 cause dominant congenital spinal muscular atrophy and hereditary spastic paraplegia. Am J Hum Genet 2013;92:965-73. doi:10.1016/.j.ajhg.2013.04.018.

[12] Rossor AM, Oates EC, Salter HK, Liu Y, Murphy SM, Schule R, et al. Phenotypic and molecular insights into spinal muscular atrophy due to mutations in BICD2. Brain 2015;138:293-310. doi:10.1093/brain/awu356.

[13] Barone V, Del Re V, Gamberucci A, Polverino V, Galli L, Rossi D, et al. Identification and characterization of three novel mutations in the CASQ1 gene in four patients with tubular aggregate myopathy. Hum Mutat 2017;38:176173. doi:10.1002/humu.23338.

[14] van Spaendonck-Zwarts KY, van Hessem L, Jongbloed JD, de Walle HE, Capetanaki Y, van der Kooi AJ, et al. Desmin-related myopathy. Clin Genet 2011;80:354-66. doi:10.1111/j.1399-0004.2010.01512.x. 
[15] O'Grady GL, Lek M, Lamande SR, Waddell L, Oates EC, Punetha J, et al. Diagnosis and etiology of congenital muscular dystrophy: We are halfway there. Ann Neurol 2016;80:101-11. doi:10.1002/ana.24687.

[16] Savarese M, Di Fruscio G, Torella A, Fiorillo C, Magri F, Fanin M, et al. The genetic basis of undiagnosed muscular dystrophies and myopathies: Results from 504 patients. Neurology 2016;87:71-6. doi:10.1212/WNL.0000000000002800.

[17] Norwood FL, Harling C, Chinnery PF, Eagle M, Bushby K, Straub V. Prevalence of genetic muscle disease in Northern England: in-depth analysis of a muscle clinic population. Brain 2009;132:3175-86. doi:10.1093/brain/awp236.

[18] Udd B. Distal myopathies--new genetic entities expand diagnostic challenge. Neuromuscul Disord 2012;22:5-12. doi:10.1016/j.nmd.2011.10.003.

[19] Carrillo N, Malicdan MC, Huizing M. GNE Myopathy: Etiology, Diagnosis, and Therapeutic Challenges. Neurotherapeutics 2018;15:900-14. doi:10.1007/s13311-018-0671-y.

[20] Bugiardini E, Morrow JM, Shah S, Wood CL, Lynch DS, Pitmann AM, et al. The diagnostic value of MRI pattern recognition in distal myopathies. Front Neurol 2018;9. doi:10.3389/fneur.2018.00456.

[21] Thompson R, Straub V. Limb-girdle muscular dystrophies - international collaborations for translational research. Nat Rev Neurol 2016;12(5):294-309. doi:10.1038/nrneurol.2016.35.

[22] Fichna JP, Maruszak A, Żekanowski C. Myofibrillar myopathy in the genomic context. J Appl Genet 2018;59:431-9. doi:10.1007/s13353-018-0463-4.

[23] Lewis-Smith DJ, Duff J, Pyle A, Griffin H, Polvikoski T, Birchall D, et al. Novel 
HSPB1 mutation causes both motor neuronopathy and distal myopathy. Neurol Genet 2016;2:e110. doi:10.1212/NXG.0000000000000110.

[24] Unger A, Dekomien G, Guttsches A, Dreps T, Kley R, Tegenthoff M, et al. Expanding the phenotype of BICD2 mutations toward skeletal muscle involvement. Neurology 2016. doi:10.1212/WNL.0000000000003360.

[25] Bernardi P, Bonaldo P. Dysfunction of mitochondria and sarcoplasmic reticulum in the pathogenesis of collagen VI muscular dystrophies. Ann N Y Acad Sci 2008;1147:303-11. doi:10.1196/annals.1427.009.

[26] Lampe AK, Bushby KM. Collagen VI related muscle disorders. J Med Genet 2005;42:673-85. doi:10.1136/jmg.2002.002311.

[27] Deconinck N, Richard P, Allamand V, Behin A, Laforet P, Ferreiro A, et al. Bethlem myopathy: long-term follow-up identifies COL6 mutations predicting severe clinical evolution. J Neurol Neurosurg Psychiatry 2015;86:1337-46. doi:10.1136/jnnp-2013-307245.

[28] Zamurs LK, Idoate MA, Hanssen E, Gomez-Ibanez A, Pastor P, Lamande SR. Aberrant mitochondria in a Bethlem myopathy patient with a homozygous amino acid substitution that destabilizes the collagen $\mathrm{VI}$ alpha2(VI) chain. $\mathrm{J}$ Biol Chem 2015;290:4272-81. doi:10.1074/jbc.M114.632208.

[29] Cetin N, Balci-Hayta B, Gundesli H, Korkusuz P, Purali N, Talim B, et al. A novel desmin mutation leading to autosomal recessive limb-girdle muscular dystrophy: distinct histopathological outcomes compared with desminopathies. J Med Genet 2013;50:437-43. doi:10.1136/jmedgenet-2012-101487.

[30] Evangelista T, Hanna M, Lochmuller H. Congenital Myasthenic Syndromes with Predominant Limb Girdle Weakness. J Neuromuscul Dis 2015;2:S21-9. doi:10.3233/JND-150098. 
[31] Klein A, Pitt MC, McHugh JC, Niks EH, Sewry CA, Phadke R, et al. DOK7 congenital myasthenic syndrome in childhood: early diagnostic clues in 23 children. Neuromuscul Disord 2013;23:883-91.

doi:10.1016/j.nmd.2013.06.002.

[32] Nakamura F, Stossel TP, Hartwig JH. The filamins: organizers of cell structure and function. Cell Adh Migr 2011;5:160-9.

[33] Atwal PS, Blease S, Braxton A, Graves J, He W, Person R, et al. Novel Xlinked syndrome of cardiac valvulopathy, keloid scarring, and reduced joint mobility due to filamin A substitution G1576R. Am J Med Genet A 2016;170A:891-5. doi:10.1002/ajmg.a.37491. 


\section{FIGURE LEGENDS}

Figure 1: Diagnostic rates in clinical and pathological subgroups

A: clinical subgroups. Clinical features with the highest diagnostic rate were childhood onset (35\%) and distal weakness (39\%). B: muscle pathology subgroups. Patients with normal/minimal changes and only general myopathic features had the lowest diagnostic hit rates (11 and $13 \%$ respectively). A pathogenic/likely pathogenic variant was found in all patients with tubular aggregates $(n=3)$. RV=rimmed vacuoles.

Figure 2: Muscle pathology and MRI Pattern of case ID40 with a pathogenic variant in BICD2.

A-D: tubular aggregates (white arrows) were demonstrated mostly in type II fibres with myoadenylate deaminase (A), NADH-TR (B), Gomori Trichrome (C) and SERCA1 (D) staining. Scale bar represents 100um.

E: lower limb MRI. In the thigh (upper part) there is fatty replacement of the muscles of the anterolateral thigh with relative sparing of semitendinosus (ST), adductor magnus (AM) and gracilis (G). In the calf (lower part) the fatty replacement involves tibialis anterior and gastrocnemius muscle with sparing of peroneus $(P)$. The muscle $\mathrm{MRI}$ images are consistent with the BICD2-MRI pattern previously reported.[12]

\section{Figure 3: COL6A-related myopathy}

A-C: ID 52 (COL6A3 c.6220G>A), D-F: ID 67 (COL6A1 c.1056+1G>A). Muscle biopsies: Oxidative stains (NADH-TR, COX, COX-SDH) showed myopathic size variation, ill-defined cores, several lobulated fibres (A, D: stars) and COX deficient fibres (B and E: stars). Scale bar represents 100um. Muscle MRI in the thigh (C, F) 
showed some typical signs of Bethlem myopathy: peripheral fat infiltration with central sparing of vasti muscles (white arrow), rectus femoris involvement with a spared area with a U- shape (white asterixis).

G-L: collagen VI-Perlecan fluorescent immunostaining in case ID 52 (G-I) and case ID 67 (J-L). Double-labeling with the collagen VI antibody MAB1944 and perlecan showed good co-localisation at the basal lamina without clear reduction compared to an unaffected control (M-O) in ID52 (G-I) and subtle reduction of collagen VI at the basal lamina in ID67 (J-L). (G-J-M, collagen VI; H-K-N perlecan; I-L-O merge).

\section{Figure 4: Muscle pathology in DES- and DOK7-related myopathy cases}

A-D: ID4, case with pathogenic variant in DES. Muscle biopsy showed marked increased variation in fibre size with fibre diameter in the range of 10-120 um and frequent round atrophic fibres. There were no cytoplasmic protein aggregates A, (H\&E). B: there was a slight disturbance of internal architecture highlighted with NADH staining (B). C-D: retrospective immunostaining for desmin revealed uneven labelling in many fibres without convincing protein aggregation (C patient, $\mathbf{D}$ control). E-F: ID 35, (compound heterozygous DOK7 pathogenic variants). Two muscle biopsies were performed at 5 and 23 years of age (E, F; NADH staining). Fibre typing is preserved in both biopsies. The first biopsy shows uneven oxidative staining in many fibres of both types. The second biopsy shows progression of these lesions into well-defined multiminicores. Scale bar represents 100um. 


\section{TABLES}

Table 1: Main clinical details of the cohort studied.

\begin{tabular}{|c|c|}
\hline Main features & $\mathbf{N}(\%)$ \\
\hline \multicolumn{2}{|l|}{ Age of onset } \\
\hline Childhood onset $(0-<10)$ & $30(30)$ \\
\hline Juvenile/adult (10-39) & $34(34)$ \\
\hline Late onset $(\geq 40)$ & $36(36)$ \\
\hline \multicolumn{2}{|l|}{ Clinical features } \\
\hline Proximal weakness & $47(47)$ \\
\hline Distal weakness & 33 (33) \\
\hline Other phenotypes & $20(20)$ \\
\hline \multicolumn{2}{|l|}{ Family history } \\
\hline Positive & $21(21)$ \\
\hline Consanguinity & $6(6)$ \\
\hline \multicolumn{2}{|l|}{ Electrophysiology $(n=84)^{\star}$} \\
\hline Myopathic & $54(64)$ \\
\hline Neuropathic & $5(6)$ \\
\hline Mixed & $13(15)$ \\
\hline Normal & $12(14)$ \\
\hline \multicolumn{2}{|l|}{ Muscle biopsy $(n=89)^{*}$} \\
\hline Normal/minimal changes & $9(10)$ \\
\hline End stage & $3(3)$ \\
\hline Neurogenic & $2(2)$ \\
\hline Myopathic total^ & $75(84)$ \\
\hline General dystrophic features & $7(9)$ \\
\hline General myopathic features & $23(31)$ \\
\hline Protein aggregation and/or rimmed & $21(28)$ \\
\hline \multicolumn{2}{|l|}{ vacuoles } \\
\hline Core-like lesions & $9(12)$ \\
\hline Tubular aggregates & $3(4)^{\prime}$ \\
\hline Nemaline bodies & $5(7)$ \\
\hline Mitochondrial features & $7(9)$ \\
\hline \multicolumn{2}{|l|}{ Muscle MRI (n=77)* } \\
\hline Normal & $12(16)$ \\
\hline Fatty infiltration $^{\dagger}$ & $65(84)$ \\
\hline Proximal & $1(2)$ \\
\hline Distal & $4(7)$ \\
\hline Both & $52(91)$ \\
\hline \multicolumn{2}{|l|}{ Number of genes already tested } \\
\hline $0-4$ & $60(60)$ \\
\hline$\geq 5$ & $40(40)$ \\
\hline
\end{tabular}

* For electrophysiology, muscle biopsy and muscle MRI percentages are calculated on the number of tests performed and retrieved.

$\wedge$ Percentages of myopathic predominant pathological features (italics) are calculated on total cases with myopathic biopsy $(n=75)$.

† Percentages of fatty infiltration distribution (italics) are calculated on total cases with abnormal MRI and both Thigh and Leg MRI scans available. 
Table 2: Comparison between patients with and without pathogenic/likely pathogenic variants for main clinical features reported.

\begin{tabular}{|c|c|c|}
\hline Feature & Positive gene result & p-value \\
\hline Childhood onset & $15 / 30(50 \%)^{*}$ & \\
\hline Juvenile/adult onset & $12 / 34(35 \%)$ & $p=0.006$ \\
\hline Late onset & $5 / 36(14 \%)^{\star *}$ & \\
\hline Proximal weakness & $14 / 47(30 \%)$ & \\
\hline Distal weakness & 13/33 (39\%) & $p=0.609$ \\
\hline Other phenotypes & $5 / 20(25 \%)$ & \\
\hline Abnormal EMG/NCS & $25 / 72(35 \%)$ & \\
\hline Normal EMG/NCS & $0 / 12(0 \%)$ & $p=0.015$ \\
\hline Abnormal MRI & $21 / 65$ (32\%) & \\
\hline Normal MRI & $\# 1 / 12(8 \%)$ & $p=0.162$ \\
\hline $\begin{array}{c}\text { Abnormal muscle } \\
\text { pathology }\end{array}$ & $24 / 80(30 \%)$ & $P=0.436$ \\
\hline $\begin{array}{c}\text { Normal muscle } \\
\text { pathology }\end{array}$ & $1 / 9(11 \%)$ & \\
\hline
\end{tabular}

\#: The patient with a normal muscle MRI was ultimately diagnosed as a myopathy mimic $(\text { FLNA })^{*}: p=0.019$ vs other age of onset, ${ }^{*} p=0.004$ vs other age of onset. 
Table 3: Prevalence of the genes identified in our cohort $(n=100)$

\begin{tabular}{cc}
\hline Gene & N of patients \\
\hline TTN & 4 \\
MYH7 & 2 \\
DOK7 & 2 \\
STIM1 & 2 \\
SEPN1 & 2 \\
DNAJB6 & 2 \\
GNE & 2 \\
COL6A1 & 2 \\
COL6A3 & 1 \\
DES & 1 \\
FLNA & 1 \\
BICD2 & 1 \\
TPM3 & 1 \\
GMPPB & 1 \\
HSPB1 & 1 \\
ORAI1 & 1 \\
ACTA1 & 1 \\
ANO5 & 1 \\
FHL1 & 1 \\
MYOT & 1 \\
MFN2 & 1 \\
VCP & 1 \\
\hline
\end{tabular}

